Rapport - Société canadienne d'histoire de l'Église catholique

\title{
L’influence de l'Église sur nos lois canadiennes
}

\section{Léo Landreville}

Volume 29, 1962

URI : https://id.erudit.org/iderudit/1007366ar

DOI : https://doi.org/10.7202/1007366ar

Aller au sommaire du numéro

Éditeur(s)

La Société canadienne d'histoire de l'Église catholique

ISSN

0318-6148 (imprimé)

1927-7075 (numérique)

Découvrir la revue

Citer cet article

Landreville, L. (1962). L’influence de l'Église sur nos lois canadiennes. Rapport -

Société canadienne d'histoire de l'Église catholique, 29, 63-71.

https://doi.org/10.7202/1007366ar

Tous droits réservés @ La Société canadienne d'histoire de l'Église catholique, 1963
Ce document est protégé par la loi sur le droit d'auteur. L’utilisation des services d'Érudit (y compris la reproduction) est assujettie à sa politique d'utilisation que vous pouvez consulter en ligne.

https://apropos.erudit.org/fr/usagers/politique-dutilisation/ 


\section{L'influence de l'Église sur nos lois canadiennes}

J'éprouve en ce moment le plus cinglant des malaises. Je connais pour une fois la douloureuse épreuve de celui qui doit être jugé. Devant un si auguste tribunal, je viens plaider coupable. Le titre même de cette causerie, "L'influence de l'Eglise sur nos lois canadiennes"; indiquerait donc que l'Eglise exercerait une influence directe sur nos législateurs et même s'immiscerait dans les jugements de Cour. Enfin, de toute apparence, ma thèse serait donc celle d'établir que l'Eglise est bel et bien entrée en politique.

Telle interprétation du sujet suscite la curiosité et c'est ce qui explique, sans doute, le nombre et la qualité de l'auditoire.

Ma culpabilité surgit du fait que je vous tiens maintenant dans mon emprise grâce à une fausse représentation. Je n'ai nullement l'intention de développer ce thème. Et si mon texte s'apparente, même de loin au sujet, ce n'est que coïncidence ou accident fortuit. Je m'empresse d'ajouter que le décousu est né du fait que je suis un juge très normal : une fois mon idée faite, je suis comble d'indécision. Prière de ne pas dire de moi ce que l'on disait de Lord Mansfield: "He was often in error - but never in doubt." On sait bien qu'en Cour de Justice si les avocats se donnent du " mon savant collègue ", c'est bien pour faire comprendre au juge qu'il ne s'agit pas de lui.

\section{Mesdames et messieurs,}

Ce dont je veux parler, c'est bien de la Loi, de quelques lois en rapport à la morale chrétienne, et du droit naturel. De prime abord disons que la Loi doit être basée sur la raison, qu'elle doit protéger la liberté de l'individu et qu'elle doit être conforme à la loi morale. Ceci n'est que de l'esthétique, toutefois, et n'est pas nécessairement la conception actuelle de la loi. La loi est dichotome et souffre deux vies. Elle a une vie politique en autant qu'elle aspire à exprimer les nécessités et les réalités d'une structure sociale. Mais elle détient également une vie technique au moment même où elle passe une élaboration scientifique aux mains des juristes.

Dans l'une ou l'autre de ces vies, se conformera-t-elle toujours à la morale et au droit naturel ? Voilà la question embarrassante et je tenterai d'y répondre par référence à quelques statuts.

Une mise au point. Même si actuellement, des trétaux politiques. on loue ou condamne les vertus et les vices de nos lois, je ne suis le porte-parole d'aucun candidat. Je m'en garde bien, car le Voters Act ontarien dit : "N'auront pas droit de suffrage, les mineurs, les idiots et les juges... dans cet ordre ! 
Un rapide coup d'œil, donc, sur les sources et l'histoire de la jurisprudence des provinces anglaises. L'acte confédératif répétait que la loi qui régit le Canada est celle qui est en vigueur en Angleterre en 1792. On transportait d'emblée le droit coutumier et le droit civil existant alors. Le premier prenait substantiellement sa source du droit canonique et en conservera des vestiges jusqu'à nos jours. Les Cours ecclésiastiques affirmaient, jusqu'au $\mathrm{XIV}^{e}$ siècle, en Angleterre, une juridiction quasi plénipotentiaire sur le sujet "pro salute animae". Car quelque offense, quelque conflit entre sujets mettait en jeu la morale chretienne et ceci etait du domaine strict de i"Eglise.

Rappelons-nous que, durant le règne des Stuarts, ni roi ni parlement ne pouvait décréter une loi à l'encontre de la morale. Et en parallèle se développait le Common Law, fondé sur la morale et le droit naturel.

On constate la puissance de cette dette dernière dans un jugement de Sir Edward Coke prononcé en 1610 où il disait :

and it appears in our books that in many cases the common law will control Acts of Parliament and sometimes adjudge them to be utterly void; for when an Act of Parliament is against common right and reason or repugnant or impossible to be performed, the common law will control it and adjudge such Act to be void. *

Quel juge, de nos jours, ne jouirait d'un tel pouvoir!

Mais il a fallu deux cents ans d'évolution avant que nous trouvions cette décision du Conseil Privé en 1842 dans la cause contre Burslem : "A court of Justice cannot set itself above the Legislature."

Du coup la loi morale et le droit naturel ne devront plus dominer la loi statutaire. Mais elle a persisté dans son existence et constitue aujourd'hui ce corps de loi appelé : Equité. Elle retient sa vigueur au sein du Common Law, entre en jeu et est invoquée dès qu'un acte législatif souffre d'ambiguïté ou de conflit avec d'autres lois. Le champ d'action est donc bien limité.

Une décision de 1958 de la Cour d'Appel de la province de Québec mérite quelques mots, car les raisons données par les juges ont fait sourciller plusieurs juristes du dehors.

La cause de Chabot contre les commissaires d'écoles de Lamorandière concernait le droit du père, un Témoin de Jéhovah, d'introduire son enfant dans une école catholique. La Loi de l'Instruction publique (Education Act) stipule bien clairement que toute commission scolaire doit prendre les mesures nécessaires afin que le cours d'étude autorisé par le comité catholique (ou protestant selon le cas) soit suivi dans chaque école. Ceci voulait-il dire que les maîtres devaient enseigner tous les sujets à tous les élèves ou seulement aux élèves catholiques? Des sept juges, cinq étaient catholiques. On décida, à six sur sept, que seuls les catholiques devaient être obligés d'étudier le sujet de religion.

Le Témoin de Jéhovah gagnait sa cause. Implicitement, le Banc affirmait la suprématie de la morale et du droit naturel sur le droit 
statutaire. Cette cause n'alla pas en appel à la Cour suprême du Canada et le mot de la fin n'est pas écrit.

Saint Thomas dit bien que toutes les lois émanent de la Loi éternelle. Etant donné que les êtres de la création divine sont seuls capables de se gouverner, ils sont les uniques sujets de la loi. C'est par extension de cette Loi éternelle que nous trouvons de nécessité chez l'homme cet ensemble de préceptes qu'est la loi naturelle.

Cette loi n'existe nulle part, disaient Cicéron, saint Thomas et, par la suite, Blackstone, si elle n'existe pas dans le cœur de l'homme. Mais elle n'y est pas à l'état nature, comme habitude. D'où la nécessité de donner corps à certains de ces concepts de la loi naturelle et celle-ci prendrait donc forme de loi positive.

Montesquieu (chapitre 11 de l'Esprit des Lois), disait à son tour :

- Les lois humaines, faites pour parler à l'esprit, doivent donner des préceptes et point de conseils. La religion, faite pour parler au cœur, doit donner beaucoup de conseils et peu de préceptes. ”

\section{Il disait encore :}

- Comme la religion et les lois civiles doivent tendre principalement à rendre les hommes bons citoyens, on voit que, lorsqu'une des deux s'écartera de ce but, l'autre y doit tendre davantage : moins la religion sera réprimante, plus les lois civiles doivent réprimer.»

Il y aurait enfin cette différence intrinsèque entre la loi naturelle et la loi positive. Dans la première il y a différence entre le bien et le mal, ce qui n'est pas toujours le cas dans la seconde.

On me pardonnera ces données élémentaires. C'est que chez nos législateurs et nos juristes, la distinction leur échappe trop souvent. Dans l'analyse et l'interprétation des lois positives, l'on s'évertue à n'y voir que des préceptes mécaniques qui ne doivent avoir aucun lien ou affinité avec le droit naturel. C'est sous cet aspect que la cause Chabot du Québec mérite admiration. Elle réaffirme que tout texte de loi, pour en déterminer sa validité, peut être passé au test de la loi morale et $d u$ droit naturel.

Admirables dames et messieurs, munis de ces pensées, je vous prie de m'accompagner. Nous examinerons trois lois qui, par leur importance sociologique, souffrent de lacunes et même d'injustice parce qu'on s'efforce d'en omettre obligation morale et droit naturel.

Il existe, à mon opinion, une brèche sérieuse dans le Labour Act ontarien, loi qui définit les droits et obligations des relations capitaltravail, gérance-employé. Absence de loi ou absence d'interprétation salutaire des lois existantes qui empêche l'observance d'une règle d'or : celle de l'Amour du Prochain. Evidemment l'on ne peut pas obliger par force de loi à aimer. Mais la loi doit exister pour que tous deux, capital et travail, n'aient pas le droit de se blesser. Si, entre individus, il est, par la loi civile et criminelle, prohibé de se faire dommages, combien plus importante et vitale pour une société est la défense pour 
une classe de faire un tort voulu et, dans certains cas, malicieux, à une autre classe. L'incompréhensible lacune légale contemporaine est bien cette inertie devant un conflit de classes. La solution s'effectue, non pas devant un tribunal impartial, mais par les principes d'une civilisation barbare et archaïque : la loi du plus fort est toujours la meilleure.

Il y a deux ans, une commission royale d'enquête en Ontario, étudiait et recevait représentations sur le statut de nos lois du travail. I.e rapnort de cette commission recommandait quelque dix-sept amendements à la loi. Sur-le-champ, pétitions, télégrammes, lettres et délégations arrivaient devant les députés pour protester contre l'ingérance gouvernementale et réclamer le statu quo. Comme résultat, on n'a rien fait, pas un seul amendement n'a été décrété. Et le rapport d'une autre commission royale prenait le chemin bien battu conduisant aux tablettes poussièreuses du libraire parlementaire.

Ce qui détermine la qualité d'un souverain ou d'un parlement c'est bien sa sagesse et son courage. Cette sagesse qui possède la perspicacité de distinguer entre les exigences légitimes d'un peuple et les demandes frénétiquement égoïstes de quelques groupes ou factions. Son courage doit se faire sentir pour les classes minoritaires en dépit des clameurs de la foule. Car, autrement, les mineurs, les aliénés, les pauvres inoffensifs et les groupes de race et de religion minoritaires ne recevraient jamais les bénéfices d'une législation que commanảe le droit naturel.

Mais ici encore le Vox populi, Vox Dei, l'opinion publique, doit faire son travail. L'influence de l'Eglise se fait sentir par ses encycliques, par les lettres et la parole de ses prélats. Et en passant, je vous rappelle la courageuse lettre pastorale de $\mathbf{M}^{\mathbf{g r}}$ Carter, évêque du SaultSainte-Marie, en marge de la grève dans les mines de Sudbury.

Dans cette sphère légale de capital-travail, laissera-t-on longtemps persister un vacuum législatif ? Je l'ignore. Mais je crois fermement que dans l'avenir nous aurons honte de cette passivité. Encore plus, à moins que ce vacuum ne soit comblé d'ici peu, l'on court le risque de voir naître des mesures rigides et extrémistes imposées par une force dictatoriale non conforme à notre système démocratique.

Mesdames, vous êtes appelées à jouer un grand rôle dans cette affaire. Vous êtes en dernière analyse la puissance derrière le trône. En fait la grève de Sudbury a été réglée grâce à une assemblée de 5,000 épouses toutes "vocales " et déterminées. Si le rôle de la mère est de tout temps la gardienne de la morale familiale, elle devient maintenant la voix du droit naturel et de la justice. Soyez optimistes, l'évolution des lois est un fait incontrôlable.

Doutez-vous que les lois changent? Je vous donne un exemple. Dernièrement, dans quelque vieux bouquin, j'ai trouvé une loi décrétée par le Parlement britannique en 1770. Je vous la lis textuellement :

THAT all women of whatever age, rank, profession or degree, whether virgin, maid or widow, that shall from and after such Act. 
impose upon, seduce and betray into matrimony, any of his Majesty's subjects by means of scents, paints, cosmetics, washes, artificial teeth, false hair, spanish wool, iron stays, hoops, high heeled shoes or bolstered hips, shall incur the penalty of the law now in force against witchcraft and like misdemeanours, and that the marriage upon conviction shall stand null and void.

Et parlant de mariage, j'en viens donc à ma deuxième étude, si saugrenu qu'en soit le sujet dans un milieu comme celui-ci. Il s'agit de la loi du divorce. Evidemment la question n'est d'aucun intérêt pour notre président et ceux du Québec puisque là, la perfection matrimoniale existe. La preuve conclusive en est bien dans le fait qu'aucun tribunal n'y a juridiction et que l'on tente maintenant de fermer la porte du Parlement fédéral à toute pétition pour divorce venant du Québec et de Terre-Neuve.

Pour nous des autres provinces, discutons-en, mais avec la prémisse bien claire que par le fait elle ne reçoit pas notre approbation. Puis-je dire en passant qu'il n'y a pas de séparation légale qui exige assentiment juridique comme dans Québec? Ici, séparation légale des époux n'est qu'un contrat formel exécuté d'un commun accord par notaire ou avocat. L'Eglise reconnaît bien que, malheureusement, certaines alliances matrimoniales échouent dans une séparation nécessaire des corps et des biens des époux pour leur propre salut.

Si l'autorité dont je tiens ce renseignement ne fait erreur (et c'est peu probable puisque c'est un évêque), le divorce est permis aux catholiques sous certaines conditions et dans certains cas. Il faut d'abord l'approbation de ses chefs religieux, puis les parties en jeu doivent donner au divorce le but ou fin de séparation légale. Il ne doit pas être obtenu en vue d'un remariage.

Je me ferai donc l'écho de nos sociologues avertis en disant que le divorce prend une étendue dangereuse au Canada. C'est le chancre de nucléus social. En Ontario, ces récentes années on constate un accroissement régulier de $10 \%$ dans le nombre des divorces accordés par nos tribunaux.

Il y a donc lieu de se poser la question : l'influence de l'Eglise et de ses fidèles doit-elle se faire sentir dans une loi qui concerne l'infime minorité des siens? Je réponds par un OUI catégorique. J'apporte à cette question l'expérience d'un homme du métier, qui prétend avoir certaines connaissances. J'ai eu à entendre au-delà de 1,800 causes de divorce. Contrairement à la coutume de mes confrères du Banc, je détiens dans mes cahiers de cour des notes olographes sur les figurants et les faits. Je les considère précieuses car, à ma connaissance, aucunes statistiques n'existent sur le sujet.

Disons d'abord qu'au Canada, il n'y a qu'une raison juridique pour l'obtention du divorce. C'est l'adultère. En Angleterre, depuis peu de temps, on a accru les raisons pour l'obtenir. Aux Etats-Unis, dans certains Etats, les raisons acceptées et acceptables frisent le ridicule. Par exemple, manger des "biscuits soda " dans le lit matrimonial constituerait une cruauté physique. 
On entend et lit depuis quelque temps de soi-disant experts sur le sujet. On soutient qu'étant donnée l'unique raison juridique du divorce, l'on est forcé, soit de commettre l'adultère, soit de perpétuer une fraude en créant des circonstances qui l'établissent au tribunal. D'où la nécessité d'élargir les cadres pour l'obtention du divorce. En outre, on allègue que le procédé légal est complexe et coûteux.

En morale, qu'en penser? Ici, je rattache le mot morale à son dérivatif latin "mores". Est-il dans l'intérêt de la société de faciliter lé dìvîúcé ?

J'affirmerai catégoriquement que son obtention est déjà très facile et pour la preuve et pour la procédure. Un relâchement additionnel ne serait que pour le bénéfice d'une très minime partie des mariages qui ont coulé. Et j'y apporte deux raisons. La première, de logique et d'expérience, la seconde, de statistiques personnelles.

Prenons le ménage qui pour quelque raison devient tendu. C'est d'abord la période de guerre froide. Silence, refus de compromettre ses idées. Puis vient la séparation de corps. Chacun va de son côté. Or je constate que, la bête humaine étant ce qu'elle est et quelque diable l'y poussant, l'homme ou la femme, dans une période d'un ou deux ans, maintes fois plus tôt, trouve consolation dans la compagnie d'une autre femme ou d'un autre homme. Les relations, à leur origine, platoniques, changent vite de caractère. Pour raison de commodité et d'économie, on transporte la brosse à dents. Vous avez là les preuves légales nécessaires. L'union s'affermit et l'insécurité de l'un ou l'autre les force à désirer mariage. L'action légale est provoquée et l'autre partie au mariage l'intente. Dans au-delà de $90 \%$ des cas, le demandeur n'y tient pas, mais il procède puisque les défendeurs défraieront les dépenses.

Deuxième raison. J'ai compilé avec autant d'exactitude que possible des chiffres. Je conclus que dans $62 \%$ des cas, les parties sont mariés depuis moins de cinq ans. J'en trouve près de $40 \%$ sans enfants, un autre $20 \%$ avec un enfant et un faible $4 \%$ où il y a trois enfants ou plus. Et voici un chiffre à retenir : $83 \%$ des cas de divorce mettent en jeu des défendeurs qui vivent actuellement en concubinage. La femme se dit "Common Law Wife "... titre qui n'existe pas en loi.

Près des trois quarts de ces $83 \%$ ont un ou plusieurs enfants. Et la raison immédiate du divorce, c'est que les défendeurs veulent légitimer l'enfant, car il ira bientôt à l'école. Sa naissance a été enregistrée sous le nom de la mère qui maintenant porte le nom de son concubin.

Des $17 \%$ des cas qui nous restent, près de $14 \%$ font voir des défendeurs qui ont actuellement cohabité plusieurs semaines ou plusieurs mois.

Il ne reste donc que près de $3 \%$ des cas où la preuve indique que l'association des défendeurs est fortuite - cas isolés, enfin cas d'un soir ... hôtel, motel... où ils ont été pris en délit. 
Donc la fabrication frauduleuse d'une preuve d'adultère ne deviendrait logiquement probable que dans ce $3 \%$ des cas. Même si l'on n'accorde pas toujours au juge une clairvoyance psychologique, il doit, de ces $3 \%$, en éliminer encore, parce qu'il doute de la véracité des témoignages.

J'espère enfin tuer ici l'expression et la croyance par trop répandues que " all divorce actions are rigged ", c'est-à-dire factices en ce qui concerne la preuve.

En ce qui a trait à la procédure, dans les actions non contestées, est-elle si complexe lorsque la moyenne du temps requis pour une action de divorce se fixe à une demi-heure en tout? D'ailleurs G. B. Shaw disait que «la procédure légale surgit du fait que... quand deux menteurs se confrontent... la vérité en jaillit !»

Et dans toute cette question que fait-on des enfants? N'ont-ils pas aucun droit et l'Etat n'a-t-il aucune obligation à leur égard ? Le bien-être social n'y serait pour rien malgré les statistiques de nos cours juvéniles?

Les Eglises catholiques et anglicanes particulièrement, par la foi de leur fidèles, doivent maintenir en échec les clameurs pour faciliter le divorce.

$\mathrm{Si}$ on me demande quelle est, selon mes constatations, une des grandes causes des faillites matrimoniales, je dirai qu'au sein de la famille se reflète ce que l'on voit à l'extérieur. C'est dans bien des cas une crise contre l'autorité. Les mariages civils et certaines cérémonies religieuses chez nos concitoyens d'autres religions omettent le serment d'obéissance de la part de l'épouse. On crée ainsi une société légale à droits égaux. Le père de famille moderne deviendrait donc soit le tyran ou la vadrouille de la famille. Evidemment, dans ces causes, c'est l'amour, la bonne volonté, enfin l'esprit purement chrétien qui manquent. On n'y trouve pas la solution facile de cette énorme dame au poignet de fer qui me disait en cour : "Si mon mari ne paie pas mon support, je vais en reprendre possession!"

Ceux qui constatent le naufrage de tant de barques matrimoniales peuvent-ils espérer, avec grand respect, des directives constamment renouvelées de la part de l'Eglise?

Mesdames et messieurs, j'en viens tardivement et brièvement au troisième point de mon pot-pourri d'idées. J'espère jouer dans la même clef - celle de la morale et de la loi.

L'un des statuts fédéraux décrété récemment a été appelé l'émule de la Magna Carta de 1216. C'est la Déclaration des Droits - le Bill of Rights passé en 1960. Je dois consacrer quelques commentaires. Dois-je dire qu'ils ne prendront pas l'allure de l'énoncé d'un cynique? "Cette Charte des droits, disait-il, s'applique à tous les citoyens du Canada - sauf à ceux qui demeurent dans une des provinces. „Il faisait allusion sans doute au fait, qu'en vertu de l'Acte de l'Amérique du Nord britannique, les provinces seules peuvent légiférer dans le 
domaine des droits de propriété et des droits civils. Qu'en plus ce champ est déjà envahi par des multitudes de lois qui doivent prendre préséance et sont intra vires dans les provinces.

Dans nos cours de justice, la tradition s'établit. Il devient "fashionable " pour les avocats de plaider le nouveau décret Déclaration des Droits. Il acquiert la popularité du Fifth Amendment américain. On serait tenté de croire même qu'il serait invoqué par les séparatistes du Québec pour obtenir leur libération du pacte confédératitif. J'cin doute.

Mais enfin ce statut et décret a-t-il quelque valeur ? Plausiblement, verrons-nous des conséquences avantageuses pour la morale et les droits du citoyen canadien? Je répondrai, ex cathedra et obiter dicta, que j'entrevois des raisons qui me portent à croire que cette Déclaration peut bien être l'instrument tant cherché pour la revendication de certains droits longtemps réclamés, surtout dans le domaine de la loi scolaire ontarienne.

Pour n'en mentionner qu'un aspect, je réfère à l'absence de la répartition des taxes scolaires par les corporations entre les écoles neutres et catholiques. C'est devenu le boulet au pied de notre système scolaire. D'où le cri constant d'un demi-siècle d'une minorité aux oreilles sourdes d'une majorité. Cette surdité est voulue pour des raisons politiques. Son importance doit nous frapper quand on voit, en 1910, 2,000 compagnies d'affaires avec chartes, et aujourd'hui, 65,000.

Les années passent, la souffrance et les sacrifices deviennent plus grands. L'espoir d'une législation équitable diminue chez plusieurs avec raison. Une expression bien connue dans les corridors du palais législatif, et dans quelque parti politique que ce soit, est la suivante : "Touchons-y pas, aux lois scolaires!"

Mais ce soir, j'oserais dire que le temps est arrivé, que l'heure est propice. Si ma pensée manque de précision, c'est à dessein. La question est matière judiciaire. Qu'il suffise de dire qu'il est temps de prendre le chemin des tribunaux et jusqu'à la cour suprême du Canada.

On excusera mon manquement à la modestie. Je réfère à la cause Vandekerchove ex versus la Corporation du canton de Middleton. Ma décision rendue en faveur des contribuables et de la Commission des écoles séparées en juin 1960, était renversée unanimement par la Cour d'appel de l'Ontario. Celle-ci, à son tour, était renversée unanimement par les juges Cartwright (qui écrivait le jugement), Fauteux, Judson et Ritchie de la Cour suprême du Canada, en décembre dernier. Cette dernière, je le dis avec orgueil, réaffirmait ma décision et les raisons apportées à son appui.

Nous devons ici une appréciation chaleureuse à l'épiscopat de London et à l'honorable Arthur Lebel, ancien collègue et avocat dans la cause. Preuve concrète de l'influence de l'Eglise et d'un lä̈cat bienveillant.

Il s'agissait de l'interprétation de certains articles du Separate Schools Act. J'apportais dans l'interprétation du statut la lumière du 
droit naturel et de l'injustice en interprétant strictement le langage de la loi. Malgré l'injustice, la Cour d'appel ontarienne refusait l'argument. Mais citons, pour terminer, ce que la Cour suprême du Canada disait :

There is ample authority for the proposition that when the language used by the Legislature admits of two constructions one of which would lead to obvious injustice or absurdity, the courts act on the view that such a result could not have been intended. That the construction adopted by the Court of Appeal results in grave hardship was fully recognized by that Court and by counsel for the respondent, who argued that the words used by the legislature did not permit any other construction.

Ce tracé conduit à la trouée de Belfort. A d'autres maintenant d'y passer, élargissant, ce faisant, les interprétations trop exiguës et injustes de nos lois scolaires.

Si parfois l'influence de l'Eglise sur nos lois paraît indescriptible et silencieuse, c'est la discrétion qui doit primer. Mais ici, le laïcat bien pensant doit s'empresser et s'imposer par son unité, sa persistance, sa force.

Si on refuse l'entrée des lois naturelles dans la loi positive, elles devront quand même continuer, comme un fantôme vampire, à extraire un sang impur d'un corps de loi positive substantiellement inadéquat et par trop inepte et malveillant.

Honi soit qui mal y pense...

Léo LANDREville, juge

Toronto, Ont. 